\title{
TECNOLOGÍAS Y CIUDADANÍA
}

\author{
ANA ABA CATOIRA \\ Profesora Titular de Derecho Constitucional \\ Universidad de A Coruña
}

Fecha de recepción: 10-11-2008

Fecha de aceptación: 26-11-2008.

Tradicionalmente, desde un pensamiento defendido como universal, se ha legitimado la separación y subordinación de las mujeres, que, de este modo, han permanecido invisibilizadas y excluidas de los ámbitos de participación, siglo tras siglo. Por tanto, sin apenas cuestionamiento crítico, englobados hombres y mujeres bajo un pensamiento androcéntrico tenido por universal y científico, se ha construido y consolidado la subordinación femenina, otorgando un papel social a los hombres y otro diferente a las mujeres fundamentado básicamente en razones biológicas y que ha determinado la posición de cada uno de los sexos.

Muy distintos y variados argumentos se han barajado para legitimar la desigualdad e inferioridad femenina. A consecuencia de ello se ha producido una injusta y discriminatoria exclusión de las mujeres de todos los procesos de toma de decisiones, de todos los momentos y situaciones en que se ha construido lo público, en los que se han establecido las relaciones de poder, para ser reducidas a su ámbito tradicional, entendido como «natural», es decir, lo doméstico ${ }^{1}$. Por ello, la histórica exclusión de las mujeres del conocimiento,

1. Es absolutamente recomendable el repaso por distintos textos filosóficos a través de AMORós, Celia: Hacia una crítica de la razón patriarcal, Barcelona, Anthropos, 1985, $2^{\text {a }}$ ed, 1991. Pongamos sólo un ejemplo sirviéndonos de la obra de KANT quien escribía: «La mujer es declarada civilmente incapaz a todas las edades, siendo el marido su curador -tutor- natural; puesto que, si bien la mujer tiene por naturaleza de su género capacidad suficiente para representarse a sí misma, lo cierto es que, como no conviene a su sexo ir a la guerra, tampoco puede defender personalmente sus derechos, ni llevar negocios civiles por sí misma, sino sólo por su representante», Antropología en sentido

Feminismo/s 12, diciembre 2008, pp. 203-228 
de los derechos y de la participación pública las ha privado de la condición de sujetos de derecho y protagonistas en la construcción de la historia, una constante que sólo se puede invertir una vez alcanzada la igualdad.

Los argumentos sobre los que se construyó la desigualdad y que se han venido transmitiendo a lo largo de los siglos, contribuyendo a socializar a generaciones enteras y sustentando todas las estructuras sociales, se difunden a través de los canales más variados. Como en cualquier otro aspecto relevante de la sociedad moderna, especialmente trascendentes a la hora de difundir aquellos $\mathrm{u}$ otros argumentos son las nuevas tecnologías de la información y la comunicación, más conocidas como TICs. En este trabajo se ha pretendido realizar una serie de consideraciones sobre el papel que pueden jugar las TICs como instrumento para construir una sociedad más integradora e igualitaria al ofrecer nuevas posibilidades de actuación y participación para las mujeres que, por ello, han de estar especialmente preparadas para ser sujeto activo en el ámbito tecnológico. En este sentido, lo cierto es que la construcción de una Sociedad de la Información igualitaria y democrática, tiene que hacerse desde la perspectiva de género, por lo cual ha de introducirse la variable género como instrumento crítico. Y, como sabemos, el género es una construcción social y cultural que, desde las diferencias entre mujeres y hombres, ha servido para legitimar la jerarquización entre los dos sexos, estableciendo qué es lo propio y característico de las mujeres y, en contraposición, qué lo es de los hombres y, en base a ello, el papel que dentro de la sociedad están llamados a desempeñar unas y otros.

Siendo conocedoras de la profunda brecha social que se abre entre mujeres y hombres, es posible tomar conciencia del potencial que ofrecen las TICs como instrumento de empoderamiento de las mujeres. A través de las tecnologías se están transformando las estructuras sociales, las formas de relacionarse, de producir, de informarse, de comunicarse, etc., y es precisamente en estos procesos de creación y transformación social donde tienen que estar representadas activamente las mujeres para que la Sociedad de la Información «universal» englobe las necesidades e intereses de las distintas identidades, de los distintos sujetos, sin que, una vez más, se tenga por universal lo propio o correspondiente al género dominante. La integración femenina en la construcción de la Sociedad de la Información supone construir una ciudadanía en condiciones de igualdad, por cuanto que su actividad social, su presencia y

pragmático, 1798, AK. VII, 209 (versión castellana de J. Gaos. Madrid, Alianza Editorial, 1991). 
visibilidad pública las empodera dejando de estar subordinadas a los hombres para disfrutar de igualdad ${ }^{2}$.

Pero sucede que la realidad nos enseña que la inclusión femenina no es un hecho, sino una mera aspiración que es necesario satisfacer ${ }^{3}$, y para ello han de identificarse cuáles son las causas de la baja participación de las mujeres en la Sociedad de la Información y cuáles son los objetivos a conseguir4.

\section{La situación real de las mujeres en la sociedad de la información: la permanente exclusión femenina}

Las mujeres han sido apartadas tradicionalmente de las tecnologías, aunque la primera programadora de la historia fuese, dato absolutamente desconocido, una mujer (Ada Lovelace) en la Inglaterra victoriana de principios del siglo XIX. Las TICs son parte de una cultura en que las decisiones y las relaciones se toman y establecen desde la perspectiva masculina, desde el patriarcado «como el sistema ideológico que ordena y clasifica el mundo de forma desigual entre hombres y mujeres bajo los principios de jerarquía, subordinación, otroriedad y opacidad, los cuales están presentes en todas las estructuras organizativas de la sociedad $»^{5}$.

En este sentido, autoras como Celia Green ${ }^{6}$, afirman que la tecnología es «predominantemente masculina y de que aunque cocinar o la jardinería utilizan tecnologías (son actividades tradicionalmente femeninas), éstas no se

2. Ciertamente, las tecnologías introdujeron cambios significativos a lo largo del siglo XX y en lo que va del XXI, pero estas mejoras, estas ventajas que introducen en todos los ámbitos, no han invertido las relaciones establecidas de marcado carácter patriarcal.

3. En este sentido, como señala CASTRO GARCIA, Carmen: «Género y TICs. Voz y presencia en la Sociedad de la Información», European Comparatives Gender and Politics, Agosto, 2005, p.3, «Las nuevas tecnologías de la información y la comunicación (TIC) tampoco son en si mismas ninguna panacea de salvación, ni ninguna utopía realizada, porque aunque facilitan el ejercicio de la palabra pública de mujeres y hombres, también continúan reproduciendo las estructuras de desigualdad y dominio».

4. En la realidad de las mujeres del día a día «Hay una pared invisible que hay que derribar, porque la noción de que es cuestión de tiempo llegar a los más altos puestos de decisión se revela falsa para las mujeres»..., esta situación de «discriminación fáctica es a veces difícil de percibir, porque una pantalla legal te dice que eres igual, que si trabajas llegarás a los puestos más altos, pero no es así» (declaraciones de María Casado, Directora del Observatorio de Bioética y Derecho de la Universidad de Barcelona en 2004, publicadas en El País, 6 de diciembre de 2004, Sociedad, p. 31).

5. Blat Jimeno, Amparo: «Informe sobre la igualdad de oportunidades educativas entre los sexos», Revista Iberoamericana de Educación, 6 (septiembre-diciembre 1994), pp. 123-145.

6. GREEN, Lelia: «Communication, Technology and Society», Sage Publications, 2002, p. 171.

Feminismo/s 12, diciembre 2008, pp. 203-228 
consideran como tecnologías porque en nuestra cultura son todas actividades femeninas (los hombres que desarrollan habilidades de jardinería profesionales tienden a verse a sí mismos como horticultores, arquitectos de paisajes, gerentes de tierra o granjeros)». En este sentido, la autora acaba diferenciando las «tecnologías de servicio» que son las relacionadas tradicionalmente con lo femenino de las «tecnologías de poder y elección» que se corresponden con lo masculino.

Así pues, desde la prehistoria, las mujeres han estado vinculadas a la tecnología (alimentación, hilar, cocinar, cuidar, etc.), pero los avances tecnológicos se consideraron desde distinta perspectiva según fuese su autor un hombre o una mujer. Por tanto, los productos inventados por los hombres se valoran por vincularse a actividades «relevantes» desde la perspectiva masculina: la caza o la guerra por ejemplo, mientras que si es la mujer quien crea e inventa, su aportación se vincula al ámbito reservado a las mujeres y se excluye del saber, del conjunto de aportaciones que construyen la ciencia y el conocimiento, por lo que se le resta importancia y toda difusión.

Aparecen así multitud de avances vinculados a actividades calificadas tradicionalmente como «femeninas», como son el cuidado del hogar y de la familia, talleres textiles, alimentación, artesanía, cuidados sanitarios, plantas medicinales, cosmética, etc., que no fueron tomadas en consideración ni se visibilizaron. Pero es que, además, tal y como acertadamente señala Rubio, «en períodos posteriores y de forma progresiva, las leyes europeas prohibieron el empleo de las mujeres en los oficios y las artes en las que habían trabajado durante los siglos anteriores. Estos espacios y estos saberes pasaron a manos masculinas de forma que el siglo XVII, marca una represión en la formación y empleo femenino» ${ }^{7}$.

Pero, la pregunta que nos ronda es ¿por qué se apartó a las mujeres de la tecnología?, ¿por qué se ha hablado y se habla tanto de la tecnofobia femenina $?^{8}$ La apropiación masculina de un espacio considerado importante por su contribución al desarrollo (desde el punto de vista masculino, desde lo que los hombres consideran importante) relegó a las mujeres a su espacio "propio» y con ellas sus aportaciones que no se consideraron importantes para la ciencia, para el desarrollo, porque para los hombres todo lo relacionado con lo doméstico carece de importancia, ni les interesa ni les preocupa.

7. RUBIO, Esther: «Rompiendo tópicos. Las mujeres en la formación profesional», http:// www.adide.org/revista, número 7, noviembre 2007, p.3.

8. Sobre la literatura feminista que trata sobre «mujeres y tecnologías», ver www.donestech.net/ca/node/1152 consultada por última vez el 08/10/2008. 
Por tanto, tras el domino tradicional que ejercen los hombres sobre las máquinas e invenciones técnicas están, una vez más, razones biológicas, causa de la exclusión femenina relegada a lo doméstico, donde aun creando tecnología no obtuvo ningún reconocimiento público. Este alejamiento se fue alimentando con tópicos sexistas como que a las mujeres no les gustan las tecnologías y no están capacitadas para aprender a utilizarlas9. Tal y como señala Carme Alemany, la tecnología doméstica se considera el pariente pobre por su relación con el ámbito doméstico, el propio de las mujeres y con menor importancia. Alemany lleva a cabo una investigación sobre los procesos de diseño, producción, venta y uso de una lavadora que muestra como las mujeres permanecen al margen del diseño de la máquina, esto es, de la toma de decisiones. Son los hombres quienes deciden y lo hacen de forma estereotipada sobre las amas de casa (son quienes las van a usar), sin tener en cuenta la opinión de las mujeres y partiendo de que son ignorantes respecto a la técnica para usarla. Y por eso dice: «Ello nos aporta un poco de luz para comprender por qué tantas mujeres y tan a menudo, de una manera intuitiva, sienten un cierto malestar hacia la tecnología, o se desentienden de ella, ya que en lugar de ser innovaciones liberadoras para las mujeres, confirman muy frecuentemente su subordinación $\gg^{10}$.

Dicho lo anterior, la pregunta que podríamos formularnos es la siguiente ¿tiene sexo la tecnología? La respuesta es evidente: es la cultura patriarcal la que, desde la construcción cultural del género, ha levantado un conjunto de barreras para las mujeres y de ventajas para los hombres que han conectado históricamente tecnología y masculinidad y sustentado la pretendida o supuesta tecnofobia femenina que forma parte de la construcción del género femenino.

Por tanto, si las TICs proponen un nuevo modelo social, las mujeres quedarán fuera del mismo si no se representa e integra equitativamente a mujeres y hombres rompiendo con las barreras estructurales y sociales, así como con

9. Los estereotipos sexistas que inundan el pensamiento que se transmite alejan la ciencia de las mujeres, así señala STANLEY, Autum: Mothers and daugthers of invention, New Jersey, Reuters, 1995, University Press, que «Conocemos pocas mujeres inventoras porque la tecnología ha sido definida excluyendo el trabajo de las mujeres. Incluso cuando el trabajo de las mujeres ha sido discutido en la historia de la tecnología, éste ha sido relegado a una posición inferior o a una posición insignificante. Y esto todavía sigue pasando»; citado por Esther RUBIO en «Rompiendo tópicos...», última consulta 18/02/2008.

10. Alemany, Carme: «Tecnología y género. La reinterpretación de la tecnología desde la teoría feminista», en BARRAL, $\mathrm{M}^{\mathrm{a}}$ José, et Al.(Eds.): Interacciones ciencia y género, Icaria, Barcelona, 1999, p. 98. 
el sentimiento de inferioridad de las propias mujeres que ven y sienten a la tecnología como algo reservado a los hombres y con la que no se sienten identificadas ${ }^{11}$.

Si planteamos esta cuestión de otro modo, nos preguntamos si sería posible analizar las TICs con perspectiva de género, es decir, analizar cómo es el acceso de mujeres y hombres a Internet (condiciones, tiempos), cuál es la formación tecnológica de mujeres y hombres y cuáles son las ventajas y los contenidos que ofrece Internet, así como el lenguaje que se utiliza y el idioma predominante.

Pero, ciertamente, aunque las aplicaciones tecnológicas ofrecen potencialidades para construir una nueva organización social, la realidad es que en la Sociedad de la Información se reproducen las mismas situaciones que en la sociedad analógica (o prácticamente las mismas), regida por estructuras y parámetros patriarcales donde las nuevas oportunidades que se nos ofrecen no se están aprovechando debidamente. Las mujeres, aun teniendo presencia y visibilidad, siguen en franca minoría, no son una fuerza con capacidad de influir, con participación efectiva en el poder establecido, en la elaboración de contenidos, en la creación y corrección del lenguaje, en las instituciones, en la docencia, etc. Se abre ante nuestros ojos con toda virulencia la brecha digital de género y, consiguientemente, un nuevo hándicap a superar,

11. Una buena prueba de la masculinización de la ciencia y la tecnología como espacio nutrido de estereotipos es el Estudio del Instituto de la Mujer y el CIDE «Trayectorias Personales y Profesionales de Mujeres con Estudios Tradicionalmente Masculinos» de 2006 que analiza la situación de las mujeres que desempeñan su labor profesional en sectores que tradicionalmente son considerados masculinos. Este estudio se divide en dos apartados en los que, por un lado, se analiza la formación profesional y, por otro, la formación universitaria. El período de estudios, las dificultades en la incorporación al trabajo, las barreras a las que se enfrentan en el trabajo y como concilian la vida laboral con la personal y familiar.

Según las conclusiones del trabajo, los estudios de formación profesional como electrónica, mecánica o fontanería son en los que existe una mayor presión y discriminación hacia la mujer y donde hay mayores dificultades para su incorporación laboral por la sobrevaloración de la fuerza física.

Dentro de los estudios universitarios con más hombres, el porcentaje de mujeres en carreras técnicas, como Informática, Caminos, Agronomía o Telecomunicaciones, es superior que aquellas que optan por ramas de formación profesional.

Asimismo, las mujeres de menos de 30 años que acaban de comenzar su trayectoria profesional, están cambiando la situación, pues cuando acceden al mundo laboral no quieren perder nada de lo conseguido y, por eso, optan por mantenerse solteras y sin hijos durante más tiempo para prolongar su formación y ser más competitivas en el mercado en el que la maternidad no juega a favor de las mujeres.

En los hombres jóvenes se aprecia un cambio, ya que cada vez se orientan más a la familia y a las tareas de casa, y van asumiendo la necesidad de compartirlas. 
la incorporación de las cuestiones de género en el ámbito tecnológico para superar todas las barreras que impiden a las mujeres tener las mismas posibilidades que los varones e integrarse plenamente en la sociedad en general.

En el logro de los objetivos antes reseñados juega un papel fundamental la introducción de la perspectiva de género como instrumento para alcanzar la igualdad y reducir la brecha social que en este caso es una brecha digital, pues la realidad nos indica cómo las políticas generales no afectan por igual a mujeres y hombres teniendo un impacto diferente al ser ignorados o conscientemente desatendidos los asuntos de género cuando se formulan ${ }^{12}$. La perspectiva de género como el instrumento válido para reconducir la situación, capaz de dar un vuelco a los datos negativos y que justifica e impulsa la adopción de las políticas de género que faciliten las condiciones que permiten el acceso, la presencia y la participación de las mujeres en la red como promotoras, creadoras, emprendedoras y directivas ${ }^{13}$.

\section{Construir la sociedad de la información desde la igualdad}

Un estudio serio de la Sociedad de la Información requiere su análisis desde la perspectiva de género, examinando la situación real de las mujeres que la integran junto a los hombres por mitad. Para ello deberá ser analizada no sólo desde los parámetros puramente masculinos, establecidos como universales, sino también desde el conocimiento de las demandas de las mujeres y de la realidad de sus derechos y su ejercicio práctico. La construcción de la Sociedad de la Información desde la perspectiva de género como medio para afrontar y reducir (incluso superar) la brecha digital, exige partir de que los contenidos y el lenguaje sexista abundan en Internet y que las mujeres entran en la Red como usuarias, pero escasamente como creadoras o productoras de tecnologías. Las nuevas tecnologías de la información y la comunicación ofrecen un abanico inmenso de posibilidades de crecimiento y mejora y constituyen un instrumento útil para la igualdad de oportunidades, pero sólo cuando

12. Veáse: Género, Constitución y Estatutos de Autonomía, FreIXES, Teresa y SEVILLA, Julia (coords.), Madrid, INAP, Colex, 2005; El tratamiento del género en el ordenamiento español. (Una visión multidisciplinar del tratamiento de la mujer en los distintos ámbitos sociales) en Palonar, A. (coord.), Valencia, CC.OO., Tirant lo Blanch, 2005 y, sobre evaluación del impacto de género: ABA CATOIRA, Ana: «Políticas de igualdad en las Comunidades Autónomas», en El Estado Autonómico. Integración, solidaridad y diversidad, VidAl, J. y Herrera, Miguel A. (coords.), Madrid, INAP, Vol.I, Colex, 2005, pp. $455-474$.

13. En este sentido, Castro García, Carmen: «Género y TIC...», cit., p. 3.

Feminismo/s 12, diciembre 2008, pp. 203-228 
las mujeres tengan acceso a las mismas en igualdad ${ }^{14}$, que estén incluidas y para ello hay que detectar las necesidades, los obstáculos, por qué no hay acceso igual, uso igual por parte de hombres y mujeres o qué debería cambiar para lograr la inclusión femenina.

Hablar de brecha digital es hablar básicamente de desigualdad en una versión actual, desde la tecnología, pero que no es más que un problema de exclusión social. La brecha digital es la separación o diferencia que existe entre las personas y los grupos en que éstas se integran, y que viene determinada por las que tienen acceso a las tecnologías y cuentan con las condiciones necesarias para utilizarlas y quienes no tienen acceso o que aun teniéndolo no cuentan con las condiciones (capacitación) para utilizarlas en su beneficio, esto es, para que les reporte alguna utilidad. La brecha como su propio nombre indica entraña desigualdad en si misma, en el acceso, utilización de los recursos y servicios, obedeciendo a diversas causas como la pobreza o escasez de recursos, las políticas actuales, la educación o la misma gestión del tiempo y operando variables como el género, la edad o la clase social.

Así, en el estudio e-España 2001 se define la brecha digital en base a cuatro elementos:

-La disponibilidad del equipo necesario, $\mathrm{PC}$ o hardware, que permita conectarse a Internet.

-La accesibilidad a Internet desde el hogar, el centro de trabajo o lugar de ocio.

- El conocimiento de las herramientas básicas para poder acceder o navegar en la red.

- La capacidad adecuada para poder hacer que la información accesible pueda ser convertida en conocimiento.

Ahondando más en la brecha digital, es posible hablar de la brecha digital de género, en cuanto que las mujeres aparecen como el colectivo o grupo dentro de la sociedad de la información con menor índice de inclusión, una brecha que lejos de disminuir sigue aumentando. Y este aumento responde a

14. «Indefectiblemente la democracia se ve afectada por todos estos procesos y cambios, que la sitúan en un nuevo contexto en permanente evolución y readaptación. Ante semejantes alteraciones la democracia no debe renunciar a sus principios básicos articulados en torno a la libertad y a la igualdad, es decir, no debe renunciar a su sustrato axiológico. Todo lo contrario: los avances del mundo digital tienen que mejorar el funcionamiento democrático al tiempo que facilitan la vida individual y social. Las exigencias de la libertad, la igualdad, el control del poder y la participación ciudadana deben seguir brillando con luz propia», FERNÁNDEZ, José J.: «La aprehensión jurídica de la democracia y el gobierno electrónicos», en Libertades, democracia y gobiernos electrónicos, Cotino, Lorenzo (coord.), Granada, Comares, 2006, p.136. 
que la integración femenina resulta difícil, más difícil que la masculina, por razones evidentes ya que estamos ante un proceso que podríamos calificar como una espiral. Y es que, si las mujeres son las más pobres y con más escasa formación, dos requisitos imprescindibles para participar (recursos y formación y capacitación) no se van a beneficiar de las ventajas o beneficios porque las relaciones de poder establecidas no invierten en personas sino en riqueza, en obtención del beneficio o productividad. La igualdad no es un objetivo prioritario, sino formar y capacitar a las personas ya integradas para que sean más rentables, por lo que si las mujeres están infrarrepresentadas se perciben como sujetos pasivos que reciben información pero que ni crean ni deciden y no se gastarán recursos en mejorar específicamente su situación.

Por eso, las políticas públicas, en su objetivo de alcanzar el desarrollo tecnológico, tienen que incluir a las mujeres, tienen que buscar la visibilización femenina dentro de la Sociedad de la Información, y esto se hace si se trabaja con perspectiva de género, que es la que hace visible las diferencias por sexo, sin atender a la variable universal que es el género masculino por entenderlo abstracto e inclusivo de hombres y mujeres.

Las políticas actuales apuestan, al menos en teoría, por la inclusión de las mujeres en la sociedad tecnológica, apuestan por terminar o al menos reducir el analfabetismo digital femenino ${ }^{15}$, y por eso nos encontramos con que la brecha digital o brecha digital de género se ha convertido en asunto de las agendas políticas, tal como se desprende del Plan Avanza que dedica un espacio a la igualdad, género y TICs, intentando establecer medidas para atajar la brecha digital y fijando un presupuesto para dicho objetivo ${ }^{16}$.

\subsection{Género y sociedad de la información en la Unión Europea}

En la construcción de la Sociedad de la Información, en cuanto sociedad que es, desarrollan un papel fundamental las personas, por tanto, las mujeres y los hombres están llamados a participar activamente. En este orden de cosas, ya en el Consejo Europeo de Viena (1998), anterior a la Cumbre de Lisboa (2000), se decidió crear un «Grupo de Alto Nivel sobre el Empleo y la Dimensión Social en la Sociedad de la Información (ESDIS)» y se aprobó una Resolución «sobre el empleo y la dimensión social de la Sociedad de la

15. Por alfabetización tecnológica se entiende el proceso de aprendizaje y de desarrollo personal dirigido a la adquisición de los conocimientos y habilidades necesarios para poder manejar una de las herramientas más importantes de nuestra sociedad: las Tecnologías de la Información y Comunicación (TICs), con Internet a la cabeza.

16. En el Plan Avanza se establecen medidas como la creación de un Observatorio de Igualdad y el Portal www.e-igualdad.net. 
Información $»^{17}$, en la que se establece que «los Estados miembros habrán de promover la aplicación de un enfoque de igualdad entre hombres y mujeres en la utilización de los medios de la sociedad de la información en las escuelas y en todos los niveles de la enseñanza y la formación».

En ese año se puso en marcha la iniciativa «e-Europe: Una Sociedad de la Información para todos» ${ }^{18}$ que se marcaba como objetivos: llevar la era digital y la comunicación en línea a cada ciudadano, hogar y escuela y a cada empresa y administración; crear una Europa que domine el ámbito digital, basada en su espíritu emprendedor dispuesto a financiar y desarrollar nuevas ideas y velar porque todo el proceso sea integrador.

En el trazado de las diez acciones prioritarias para alcanzar los objetivos anteriores como colectivos beneficiarios están la juventud y las personas con discapacidad, pero aparecen las mujeres específicamente.

Más adelante, tanto en la Cumbre de Lisboa, como en los Consejos Europeos de Niza y Santa María de Feria (2000) se remarca la dimensión social de la sociedad de la información y el papel que las TICs están llamadas a desempeñar con el objetivo de alcanzar la igualdad entre mujeres y hombres en la Unión Europea. No obstante, la igualdad, las cuestiones de género, siguen sin ocupar un lugar propio en las agendas, aparecen como algo colateral, pero sin entidad suficiente, y así en la estrategia «e-Europe 2002» se objetiva la lucha contra la exclusión digital centrada en las personas con discapacidad, pues «se trata de fomentar la participación de las personas discapacitadas en el ámbito de las tecnologías de la información, así como de cualquier persona que no esté en condiciones de beneficiarse plenamente de la sociedad de la información».

En el marco de esta estrategia se publica una nueva resolución relativa a la «integración social mediante las tecnologías electrónicas ${ }^{19}$, que incide en la utilización de las tecnologías para fomentar la integración y la participación, hablando de incorporar la igualdad de sexos en las políticas de integración tecnológica, para que aumente el número de mujeres que aprovechen las posibilidades de empleo y formación en la sociedad de la información. Además,

17. Resolución del Consejo y de los representantes de los Gobiernos de los Estados Miembros, reunidos en el seno del Consejo de 17 de diciembre de 1999 sobre el «empleo y la dimensión social de la sociedad de la información» (2000/C8/01).

18. Comunicación de 8 de diciembre de 1999, relativa a una iniciativa de la Comisión para el Consejo Europeo extraordinario de Lisboa de 23 y 24 de marzo: «e-Europe: Una sociedad de la información para todos» (COM 1999 687).

19. Resolución del Consejo de 8 de octubre de 2001, relativa a la integración social mediante las tecnologías electrónicas: aprovechar las oportunidades de integración social que brinda la sociedad de la información (2001/C 292/C). 
se aprobó otra Resolución sobre «mujeres y la ciencia» animando a promover el papel de las mujeres en la ciencia y tecnología ${ }^{20}$.

Ya en 2002 en el Consejo Europeo celebrado en Sevilla se acordó un nuevo plan de acción «e-Europe 2005» entre cuyos objetivos destaca la inclusión digital como accesibilidad para las personas discapacitadas, pero, sin concretar, una vez más, la capacitación y accesibilidad de las mujeres.

De los documentos de trabajo elaborados por el Grupo de Alto Nivel destacamos «Construyendo la Sociedad del Conocimiento: interacciones entre capital social y humanos» $»^{21}$, que concreta las barreras existentes para la implantación de la sociedad de la información y dedicando un apartado a la dimensión de género en el análisis de la brecha digital. La importancia de este documento se proyecta en que a partir de él derivarán otros que abordarán el género con más decisión, como la Resolución del Consejo sobre el capital social y humanos- Formación del capital social y humano en la sociedad del conocimiento: aprendizaje, trabajo, cohesión social e igualdad entre hombres y mujeres ${ }^{22}$; el Informe del Parlamento sobre las mujeres en la nueva sociedad de la información ${ }^{23}$ y la Resolución del Consejo sobre acceso y participación igualitarios de hombres y mujeres en la sociedad del conocimiento para el crecimiento y la innovación ${ }^{24}$.

Por vez primera, se profundiza en cuestiones de género, visibilizando las diferencias entre mujeres y hombres dentro de la sociedad de la información: el menor acceso, las diferencias en el uso y la participación desequilibrada en la formación tecnológica o en las empresas tecnológicas. Pero no sólo se muestra la desigualdad o el desequilibrio, sino que se incorpora la perspectiva de género en cuanto que se presentan medidas paliativas o correctoras que la Comisión y los Estados miembros han de desarrollar para lograr: la inclusión de las mujeres en todos los sectores TICs; fomentar la participación femenina en los estudios técnicos; aumentar la participación femenina en la educación superior en tecnologías; impulsar la formación tecnológica; la

20. Resolución del Consejo de 26 de junio de 2001, sobre «la ciencia y la sociedad y sobre las mujeres y la ciencia», (2001/C 199/01).

21. Commission staff working paper: «Building the Knowledge Society: Social and Human Capital Interactions». 28.5.2003.SEC (2003).

22. Resolución del Consejo de 15 de julio de 2003, sobre el capital social y humanoFormación del capital social y humano en la sociedad del conocimiento: aprendizaje, trabajo, cohesión social e igualdad entre mujeres y hombres (2003/C 175/02).

23. Informe sobre las mujeres en la nueva sociedad de la información (2003/2047 (INI)), 15 de julio de 2003. Final A5-0279/2003.

24. Resolución del Consejo de 27 de noviembre de 2003 sobre acceso y participación igualitarios de hombres y mujeres en la Sociedad del Conocimiento para el crecimiento y la innovación (2003/C 317/03).

Feminismo/s 12, diciembre 2008, pp. 203-228 
creación de nuevas oportunidades de empleo; promover el uso de las TICs en la lucha contra la violencia de género; lograr la inclusión femenina en todos los espacios públicos e integrar la perspectiva de género en todas las políticas públicas.

En esas mismas fechas se celebró en Atenas la Conferencia Europea sobre «Género y Sociedad de la Información» aunque pocas de las propuestas que surgieron han dado resultados.

El Reglamento 808/2004, relativo a estadísticas comunitarias de la sociedad de la información, adoptado por el Parlamento y el Consejo, exige a los Estados presentar estadísticas anuales relacionadas con la sociedad de la información en dos módulos: empresas y personas y hogares, por lo que se ofrecerán datos estadísticos por sexo. En este sentido, se elaboran las encuestas del INE «Encuesta de uso de TIC y comercio electrónico en las empresas» y, «Encuesta sobre Equipamiento y Uso de las Tecnologías de la Información y la Comunicación en los hogares», apareciendo la variable sexo en los resultados.

En 2005 se procede a la revisión de la Estrategia de Lisboa, de donde surge la Comunicación «Retos para la sociedad de la información europea con posterioridad a $2005 »^{25}$, entre los que figura la «e-inclusión» al considerarse prioritario el acceso de todas las personas a las tecnologías y otra Comunicación ${ }^{26}$ que contiene un conjunto de directrices diseñadas para fomentar el empleo. Sin embargo, una vez más, en ninguno de los documentos se hacer mención alguna a la situación específica de las mujeres tal como se hace con las personas minusválidas o las ancianas.

Un nuevo paso viene dado con la estrategia «i2010: la sociedad de la información y los medios de comunicación al servicio del crecimiento y el empleo ${ }^{27}$ con tres prioridades propuestas destacando, una vez más, el objetivo de la inclusión social. Del primer informe anual surgido de esta estrategia, en el que se reúnen las actuaciones que la Comisión impulsará en 2006 y

25. Comunicación de la Comisión al Consejo, al Parlamento Europeo, al Comité Económico y Social Europeo y al Comité de las Regiones de 19 de noviembre de 2004: «Retos para la sociedad de la información europea con posterioridad a 2005», (COM (2004) 757).

26. Comunicación del Presidente de la Comisión, de 12 de abril de 2005, que incluye una propuesta de decisión del Consejo relativa a las directrices para las políticas de empleo de los Estados miembros: Orientaciones generales de política económica (OGPE) y las propuestas de directrices de empleo para el período 2005-2008.

27. Comunicación de la Comisión de 1 de junio de 2005, al Consejo, Parlamento Europeo, Comité Económico y Social Europeo y al Comité de las Regiones, (COM (2005) 229 final). 
2007 entre las que figuraba la Conferencia Ministerial en Riga celebrada en junio de 2006, en la que se marcó como objetivo el equilibrio de género que, como siempre, tampoco se concretó. Con lo que, tal como se comprueba, el género no ocupa un lugar propio y específico en la Unión Europea sino que aparece como una cuestión más dentro de las estrategias o políticas de inclusión social.

Ahora bien, sí que la UE ha diseñado algunas estrategias en materia de igualdad entre mujeres y hombres como es el «Plan de Trabajo para la igualdad de oportunidades entre las mujeres y los hombres 2006-2010» $»^{28} \operatorname{con} 6$ áreas de actuación de la UE a favor de la igualdad: independencia económica, conciliación, representación paritaria, erradicación de la violencia de género, eliminación de estereotipos sexistas y promoción de la igualdad de género fuera de la UE.

\subsection{Género y sociedad de la información en España}

Hasta llegar al Plan Avanza varias han sido las actuaciones estratégicas puestas en marcha para impulsar la Sociedad de la Información en línea con las propuestas europeas. Desde «INFO XXI», pasando por «España.es» e «Ingenio $2010 »^{29}$, transcurrieron varios años en los que no se alcanzaron los objetivos propuestos por los diferentes gobiernos.

El Plan Avanza se estructura en cinco áreas de actuación siendo la de «Ciudadanía Digital» la que busca la inclusión de las mujeres, puesto que tal como se dice $« \ldots$ en los instrumentos concretos de ejecución de las medidas se introducirán previsiones dirigidas a la incorporación de las mujeres a la Sociedad de la Información y a evitar la discriminación por razón de sexo, y un seguimiento de los indicadores de ejecución para, en su caso, adoptar medidas tendentes a disminuir la brecha digital de la mujer». Dentro del área de actuación se contempla el «Género como actuación para la inclusión de la ciudadanía 2006-2008» con una dotación de 54,6 millones de euros en el conjunto de los 9,3 millones correspondientes al área.

28. Comunicación de la Comisión al Consejo, al Parlamento Europeo, al Comité Económico y Social Europeo y al Comité de las Regiones: «Plan de trabajo para la igualdad entre las mujeres y los hombres, 2006-2010». COM (2006) 92 final.

29. Para hacer un recorrido por las diferentes propuestas ver mi trabajo ABA CATOIRA, Ana: «La acción pública en la construcción de la Sociedad de la Información» en CotinO HUESO, L. (coord.): Libertades, democracia y gobierno electrónicos, Granada, Comares, 2005, pp. 301-321.

Feminismo/s 12, diciembre 2008, pp. 203-228 
Por tanto, nos encontramos con el planteamiento claro, por fin, de un doble objetivo: alcanzar la igualdad de género en la sociedad de la información y aprovechar el instrumento tecnológico para alcanzar el objetivo anterior ${ }^{30}$.

La Ley de Igualdad (LOIE) ${ }^{31}$ contempla la incorporación o inclusión de las mujeres en la sociedad de la información a través de políticas públicas. En este orden de cosas, el art. 28 se dedica a la sociedad de la información estableciendo que: 1. «Todos los programas públicos de desarrollo de la Sociedad de la Información incorporarán la efectiva consideración del principio de igualdad de oportunidades entre mujeres y hombres en su diseño y ejecución. 2. «El Gobierno promoverá la plena incorporación de las mujeres en la Sociedad de la Información mediante el desarrollo de programas específicos, en especial, en materia de acceso y formación en tecnologías de la información y de las comunicaciones, contemplando las de colectivos de riesgo de exclusión y del ámbito rural. 3. El Gobierno promoverá los contenidos creados por mujeres en el ámbito de la Sociedad de la Información. 4. En los proyectos del ámbito de las tecnologías de la información y la comunicación sufragados total o parcialmente con dinero público, se garantizará que su lenguaje y contenidos sean no sexistas».

El art. 30 dedicado al «desarrollo rural» también incide en las políticas públicas de igualdad de oportunidades en el acceso a las tecnologías mediante el uso de políticas y actividades dirigidas a la mujer rural.

En la Ley de Igualdad no se olvida un aspecto imprescindible como es la dotación presupuestaria, sin la cual toda previsión queda en mera letra escrita, ya que en sus Disposiciones Adicionales se prevé que la Ley de Presupuestos Generales del Estado constituya un Fondo especial en materia de sociedad de la información e igualdad, con tres millones de euros anuales para 2007, 2008 y 2009.

Visto lo anterior, indicaremos los ámbitos a trabajar por parte de las administraciones y, cómo no, por parte del sector privado para atajar la separación

30. Así, según la Orden de Bases ITC/2234/2006 de 5 de julio, se realizarán convocatorias de concesión de ayudas para realizar actuaciones que impulsen la igualdad en la sociedad de la información. Las actuaciones objeto de ayuda dentro de la convocatoria de ayudas 2008 «Igualdad de Género en la Sociedad de la Información» son las siguientes: estudios, evaluación de las políticas públicas y análisis de buenas prácticas en el uso de las TICs como herramienta para la igualdad de género, presencia, usos y demandas de las mujeres en relación con las TICs, realizar experiencias piloto para promover la incorporación, el uso, el autoempleo o el teletrabajo, promocionar la capacitación de la mujer para la gestión telemática, experiencias de demostración de soluciones para paliar la violencia y situaciones de riesgo o la creación, mejora e impulso de redes digitales de mujeres y participación.

31. Ley Orgánica 3/2007, de 22 de marzo, para la Igualdad Efectiva de Mujeres y Hombres. 
entre mujeres y hombres que nos ofrecen todos los indicadores de la Sociedad de la Información.

\section{Puntos débiles o ambitos a trabajar}

En este último apartado partimos de la que constituye la primera de nuestras conclusiones: las TICs no son neutrales respecto al género, pues, en si mismas, perpetúan la producción y reproducción de estereotipos sexuales presentes en nuestra forma de pensar y en nuestro comportamiento. Por tanto, la tecnología hay que tenerla en cuenta como variable cuando se analiza una sociedad, pues existe una profunda brecha social motivada por las tecnologías que se abre entre las personas que tienen acceso y las que permanecen excluidas del ámbito tecnológico.

La primera tarea a abordar será reconocer las barreras existentes que determinan que aunque las mujeres y hombres participen a través de las TICs, lo hagan de forma desigual, pues sólo tras detectarlas será posible plantear las propuestas de cambio que inviertan las cifras de la menor participación femenina. Así, por ejemplo, un obstáculo profundamente arraigado y difícil de erradicar son los patrones tradicionales de socialización o educación de nuestras niñas y niños cuya superación posibilitará el necesario cambio de roles y estereotipos (imágenes que se difunden, las creencias que se sostienen como científicas, los perfiles, etc.). Sólo tras este ejercicio de crítica, de revisión de las estructuras establecidas, la sociedad estará en condiciones de caminar hacia otra de las afirmaciones que sostenemos en este trabajo y que proyecta la realidad deseable. Nos estamos refiriendo a las TICs como herramienta de lucha por los derechos de las mujeres, no sólo en el ámbito de la participación política, sino también en el ámbito del trabajo, para que la conciliación sea una realidad, o incluso como vía de lucha contra la violencia de género, todo lo cual implica y requiere que las mujeres estén capacitadas para dominar y usar la tecnología en su beneficio ${ }^{32}$.

Evidentemente, esta cuestión tan compleja, lograr la integración femenina en el ámbito tecnológico, no se solventa con señalar un par de elementos o factores que condicionan las diferencias entre sexos o con indicar un par de ámbitos a trabajar. La inalcanzada integración tecnológica o inclusión femenina en la Sociedad de la Información responde a causas muy profundas y de lo más diverso, con tradición histórica muchas de ellas, y que van desde

32. En este sentido, reseñar la campaña «Dominemos la tecnología» iniciativa del PARM del APC, red mundial de mujeres que apoyan el trabajo en red para el cambio social y el empoderamiento de la mujer a través de TICs, fundada en 1993.

Feminismo/s 12, diciembre 2008, pp. 203-228 
el elemento educacional hasta el elemento más material en cuanto que hablamos de recursos y medios disponibles.

\subsection{La integración femenina: acceso, uso, diseño y producción}

El primer factor que determina la integración femenina es el elemento educacional o alfabetización tecnológica de las mujeres. Ya se señalaba, en líneas anteriores, cómo la sociedad patriarcal alejó a las mujeres del conocimiento en general y de la técnica en particular ${ }^{33}$. Esta realidad se comprueba en todas las vías de transmisión del conocimiento como son la escuela o la universidad, y tanto en los contenidos, en los materiales, en el lenguaje como en los comportamientos. Y cómo la socialización nos marca y configura nuestras actitudes y pensamientos, es la causa principal de que, en la actualidad, nos encontremos con que las mujeres están escasamente representadas en las carreras universitarias relacionadas con la tecnología, tanto como docentes como alumnas, que se dé una alarmante escasez de mujeres en los espacios públicos relacionados con la tecnología, tanto en la industria como en las instituciones y que, aún, en pleno siglo XXI nuestra sociedad continúe reproduciendo estereotipos sexistas, modelos masculinos en este ámbito, un lenguaje que minusvalora e invisibiliza a las mujeres y unos contenidos repletos de sexismo $\mathrm{y}$ violencia contra las mujeres ${ }^{34}$.

En el Estudio «Políticas de apoyo al software libre y abierto. Género: Informe de conclusiones» ${ }^{35}$, se denuncia cómo la infrarrepresentación femenina, la inferioridad de los sueldos y cargos femeninos, el alejamiento femenino de la tecnología, están motivados por «el entorno hostil que deben afrontar y no, como se suele decir, porque los trabajos técnicos no les interesan», una

33. Sobre educación consultar SUBIRATS, Marina: «De la enseñanza segregada a la coeducación» en Instituto de la Mujer. Mujer y Educación, Madrid, Instituto de la Mujer, 1985 pp. 9-21 y «Niños y niñas en la escuela. Una exploración de los códigos de género actuales», Educación y Sociedad, 4 (1990), pp. 91-100. Asimismo, la sección dedicada al «derecho a la educación» de la obra colectiva Las Mujeres Parlamentarias en la Legislatura Constituyente, Sevilla, Julia (dir.), Madrid, Cortes Generales, Ministerio de Presidencia, 2006, pp. 73-75.

34. La exclusión académica, quizás por mi profesión puede resultar más cercana, permite hablar de tres tipos de segregación: horizontal referida a la situación de las mujeres en aquellas carreras y especialidades consideradas de menor importancia o menor mercado; vertical, que relega a las mujeres a puestos más precarios y de menos relevancia; y temporal, impidiendo la promoción de las mujeres o haciéndola más lenta que la de los hombres. AA.VV. Informe Etan. La política científica de la Unión Europea. Promover la excelencia mediante la integración de la igualdad entre los géneros, Bruselas, Comisión Europea, 2001.

35. Estudio de la Universidad de Cambridge, sobre la situación en Europa, EE.UU e India. 
afirmación que se confirma con la mayor participación femenina en las comunidades de software libre y el retraso en el tiempo, ya que «las mujeres entran más tarde», pues la edad media es de 12 años para los hombres que se eleva a los 14,5 de ellas con lo que nos encontramos con una formación femenina más «autodidacta», concluyendo que esta situación se normaliza visibilizando a las mujeres en los ámbitos relacionados con las tecnologías y rompiendo de una vez por todas con el «techo de cristal».

El acceso tecnológico requiere, pues, una adecuada formación y capacitación que choca frontalmente con la tradicional socialización de las niñas cargada de un imaginario discriminatorio que transmite un mundo estereotipado, un universo apropiado por los hombres donde las mujeres no están presentes como protagonistas o como sujetos activos, lo que ha impedido introducir los temas e intereses propios de las mujeres, que han entrado en el universo masculino que no integra la perspectiva femenina.

Sin embargo, el acceso e integración de las mujeres depende de otros factores como el tiempo, lo que nos lleva a referirnos a la organización de las funciones que nuestra sociedad atribuye a las mujeres y a los hombres que, por descontado, libera a los hombres de determinadas actividades ligadas tradicionalmente con el espacio doméstico y les otorga mucho más tiempo para formarse, capacitarse y participar a través de las TICs.

Por tanto, resulta clave abordar el desarrollo tecnológico con perspectiva de género, potenciando la participación de las mujeres en las distintas fases del proceso, para que dejen de ser exclusivamente usuarias, para convertirse en productoras de tecnología y en gestoras del conocimiento. Desde una perspectiva de género se comprueba cómo estos son dos de los sectores en los que es manifiesta la infrarrepresentación femenina y que, por tanto, constituyen factores clave para la exclusión femenina de la Sociedad de la Información.

La importancia de este factor se manifiesta en la realidad de que las mujeres poseen un poder de influencia mucho menor que el de los hombres que conlleva una menor posibilidad de decidir, de informar, de orientar. El acceso como creadoras permitirá hacer que las TICs se presenten con una cara más amable y apetecible para las mujeres sin estar presididas por los parámetros patriarcales, lo que atañe al diseño, contenidos, lenguaje empleado, todo lo que permite una identificación de las mujeres con las TICs.

Con esta implicación femenina como creadoras de tecnología se podrá cambiar la realidad tecnológica que nos dice que las mujeres que han accedido a la Sociedad de la Información mantienen una actitud bastante crítica con el diseño ofrecido que no facilita el uso. Asimismo, las usuarias denuncian que la información en la Red está falta de calidad y de organización y que 
tampoco resulta muy fiable, y que los servicios no funcionan correctamente, sufriendo interrupciones de las conexiones.

La dominación de la tecnología por parte de las mujeres facilitará su participación en diversos ámbitos de la sociedad, como, por ejemplo, en el espacio laboral que presenta una inserción femenina menor donde juega un papel determinante el elemento tecnológico. El porcentaje inferior de mujeres respecto a los varones que acceden al mercado laboral se agrava en la Sociedad de la Información donde las relaciones sociales y económicas se rigen desde nuevas coordenadas definidas desde las competencias y capacidades tecnológicas, por lo que accederán al mercado de trabajo en las mejores condiciones aquellas personas que sean rentables en un sistema económico basado en el conocimiento. Así lo anterior, el mayor y mejor número de oportunidades será para las personas que están capacitadas y formadas en tecnologías, y que, por tanto, sabrán aplicarlas para producir en el nuevo sistema.

Este criterio conforma la sociedad de la información, diferencia socialmente a sus integrantes integrando o excluyendo, en definitiva, jerarquizando. En este punto, las mujeres están por detrás de los hombres en lo que se refiere a la incorporación a tareas profesionales y asunción de cargos de dirección y, en consecuencia, a la remuneración o sueldos. Por tanto, las oportunidades no están igual repartidas entre mujeres y hombres. Para que las cosas puedan cambiar y las mujeres puedan acceder en las mismas condiciones que los hombres deben acceder a la tecnología y obtener capacitación, para no quedar excluidas del reparto de las tareas productivas y de los puestos de más alto nivel en la economía de la información. Tradicionalmente las ocupaciones llamadas femeninas son ajenas a las nuevas tecnologías y, tradicionalmente, la orientación profesional de las mujeres no fue hacia el sector tecnológico.

Este diferente acceso a las tecnologías no puede considerase, en ningún caso, una cuestión menor, pues conlleva el ansiado acceso a la información, indispensable para ejercer cualquier actividad en las mejores condiciones y participar activa y responsablemente en cualquier ámbito. Por tanto, las nuevas tecnologías, determinantes del nuevo contexto social, ofrecen un nuevo perfil de la ciudadanía. Y es aquí donde se abren nuevas oportunidades para las personas que históricamente se han visto privadas de la posibilidad de participar en la construcción de lo público como es el caso de las mujeres que, gracias a un buen y provechoso uso tecnológico pueden participar más y mejor. 


\subsection{Presencia femenina en internet}

A pesar de los obstáculos estructurales que determinan la menor presencia femenina en la Red, lo cierto es que las mujeres cada vez están más conectadas y participan más activamente, ya sea a través de listas de correo, foros de debate, creando páginas web o construyendo redes electrónicas. Lo realmente significativo no es el acceso a Internet sino la cantidad y calidad del acceso, es decir, la banda ancha, la alta velocidad, la localización de información provechosa y rentable, etc., así como la creación de tecnología y la elaboración de contenidos. Es aquí, en este nuevo contexto político donde nos encontramos con un nuevo perfil de ciudadana activa, tecnológicamente hablando, que detenta poder de influencia que revierte en una orientación de género en Internet. Esta actividad femenina es la que permite que las TICs sean aprovechadas como una fuente de recursos inagotables, como un poderoso instrumento de empoderamiento femenino a través de las redes virtuales ${ }^{36}$. Nos referimos a las webs creadas por mujeres o las redes electrónicas que pueden ayudar al cambio social al ser nuevos espacios de participación social. Por tanto, nos encontramos ante nuevas representaciones de la figura femenina que no se reduce a informaciones sobre la belleza, cuidado del hogar y de la familia, sino que presentan a mujeres activas que luchan contra las desigualdades y por la defensa de los derechos de las personas o a mujeres profesionales que tienen poder de influencia y decisión.

Las redes electrónicas, los blogs, son dos canales de participación femenina en la Red que contribuyen a construir la igualdad desde la perspectiva de género y que, por tanto, permiten avanzar hacia una sociedad democrática. Las redes electrónicas construyen espacios de comunicación solidarios, donde se informa sobre temas diversos y se diseñan estrategias comunes de actuación. Los blogs como diarios o cuadernos de bitácora digitales incrementan la participación dentro del ciberespacio coadyuvando a la construcción de una ciudadanía activa.

36. No son pocas las referencias que se hacen a las mujeres como pioneras en el tejido de redes, si bien, al principio, redes tejidas con fibras vegetales, ya desde las más antiguas tribus primitivas y posteriormente a lo largo de toda la historia. Ver, GUIL BOzAL, Ana: «Mujeres, Universidad y cambio social: tejiendo redes» en El cambio social en España. Visiones y retos de futuro, Sevilla, Centro de Estudios Andaluces, 2006, p. 6. «Si nos acercamos a la historia más reciente, en la primera década del siglo XIX Ada Byron, fue pionera en el campo de la informática con sus aportaciones a la «máquina analítica», sus programas para cálculos matemáticos avanzados, sus trabajos sobre el primer lenguaje en la historia de la computación y sobre la matemática de la función cerebral».

Feminismo/s 12, diciembre 2008, pp. 203-228 
La apropiación de la Red por las mujeres supone transformar la información contenida, las ofertas culturales, los recursos ofrecidos, la imagen de la mujer y el consiguiente cambio de los roles estereotipados establecidos. Esta incorporación y participación femenina supone el enriquecimiento a través de otras perspectivas y visiones del mundo distintas a las establecidas como universales y permanentes ${ }^{37}$.

Un ejemplo de lo que estamos diciendo es la lucha contra la violencia en la Red y las tecnologías en general. Las tecnologías contribuyen a la violencia, a la perpetuación de una cultura machista contra las mujeres. Es algo que se evidencia cuando nos conectamos a la Red, lo que se transmite por Internet (contenidos, lenguaje, imágenes) o cuando se manejan aplicaciones tecnológicas como los videojuegos, que están imbuidos de violencia simbólica contra las mujeres o de violencia explícita en cuanto que perpetúan una representación sexista y desequilibrada de lo masculino y lo femenino.

Los videojuegos son un claro exponente de lo que estamos diciendo, en la representación que hacen de las mujeres, en que su diseño, desarrollo y producción está dominado por los hombres. Las relaciones de género también tienen presencia en este ámbito y su entendimiento correcto implicaría que las mujeres entrasen de forma activa en su creación, diseño y consumo.

En este orden de cosas, analizaremos brevemente el mundo de los videojuegos como exponente de la discriminación sexista por ser un ámbito en el que domina la violencia, la competencia, la agresión y la subordinación femenina. Las niñas no encuentran una oferta que responda a sus gustos e intereses como sí encuentran los niños, pues la habilidad, la destreza o las aventuras que responden a sus demandas, no encuentran respuesta en la industria. ¿Por qué?, no venderían ante un mercado extenso como es el femenino, o es simplemente que los creadores de software son hombres y diseñan conforme a sus gustos masculinos que universalizan en el mercado. La respuesta siempre es la misma: el patriarcado domina también el mercado de los videojuegos, que son un potente instrumento de socialización, y que, bien empleado, contribuiría a fomentar la igualdad y a integrar a las mujeres en las nuevas tecnologías.

37. La relación entre mujeres y nuevas tecnologías abre ante nuestros ojos unos objetivos muy deseables en la lucha por la igualdad efectiva, pues permite introducirse en «todos aquellos campos en los que el proceso tecnológico presenta un sesgo sexista por el cual se excluye a las mujeres de las posiciones de poder dentro de la tecnocultura», porque no podemos olvidar que la tecnología -aspiradoras, lavadoras y demás electrodomésticos- ha sido ya utilizada para aumentar el confinamiento de las mujeres dentro del hogar, y no para liberarlas: GuIL BozAL Ana: «Mujeres, Universidad y cambio social: tejiendo redes», art. cit., p. 7. 
La relación entre los videojuegos y el género se podría calificar cuando menos de polémica si partimos de que no se trata de un mero entretenimiento y sí de un poderoso instrumento de socialización. Lo más sorprendente es que no existe ninguna regulación sobre sus contenidos, que transmiten mensajes estereotipados sobre lo masculino y lo femenino, y no se utilizan como instrumento de construcción de identidades imbuidas de valores democráticos. Por tanto, los videojuegos son una forma de exclusión social, e incluso, en el peor de los casos, de una nueva modalidad de violencia de género. El vacío legal en el que se mueve esta poderosa industria pone de manifiesto la inexistente voluntad de las Administraciones por regular este ámbito, quedando como solución, aunque insuficiente, los códigos de autorregulación de la industria del software de entretenimiento. La situación actual pone de manifiesto un incumplimiento de la Convención sobre Eliminación de todas las Formas de Discriminación (art. 5) o la Convención sobre los Derechos del Niño y la insuficiencia del Código Pan European Game Information (PEGI) que en 2003 se elaboró como código de autorregulación de la industria europea de software, etiquetado y publicidad de los videojuegos y con validez en dieciséis países. Asimismo, tampoco parece una situación en consonancia con la jurisprudencia del TJCE, que el 14 de octubre de 2004 falló a favor de las autoridades alemanas respaldando la prohibición de la explotación comercial de aquellos juegos de entretenimiento que simulen acciones homicidas en contra de la dignidad.

De un Estudio elaborado por el Centro de Investigación y Documentación Educativa (CIDE) y el Instituto de la Mujer que analiza 25 de los juegos más vendidos, se concluye que los principales consumidores están seguros de que la representación de estereotipos sexistas y los valores discriminatorios no les afectan en su vida cotidiana.

Del documento «La diferencia sexual en el análisis de los videojuegos» presentado en las III Jornadas de Investigación sobre mujeres en la Educación, se desprende un interesante análisis sobre los estereotipos transmitidos en los videojuegos. Así, de 1814 personajes analizados un 64\% son hombres, un $17 \%$ mujeres y un $19 \%$ no humanos. Los pocos personajes femeninos aparecen hipersexuados y los masculinos hipermasculinizados, 9 de cada 10 mujeres de videojuego tienen un cuerpo atrayente y un $67 \%$ son demasiado delgadas y extremadamente desproporcionadas y llevan ropa semitransparente o insinuante. Un $87 \%$ de estos personajes femeninos tienen una cara angelical -perpetúan el mito de la eterna adolescente-. Aparece un nuevo personaje femenino que es una imitación del masculino, el físico, el rol atribuido.

Asimismo, según datos de la Asociación Española de Distribuidores y Editores de Software de Entretenimiento (ADESE) relativos a 2005: el 78\% de 
los niños y niñas entre 11 y 16 años juegan con videojuegos, por lo que la gravedad de la situación es manifiesta. La ausencia de regulación específica y que el Gobierno español apueste por la autorregulación permite que abunden los asesinatos, las agresiones, violaciones, la esclavitud o torturas, por lo que se perpetúan los estereotipos (discriminación), la prostitución (papel secundario de las mujeres), la invisibilización femenina y la caracterización de las mujeres como objetos al servicio de los hombres.

La integración tecnológica, además del poder de cambiar las cosas, permitirá otras ventajas que reportarán notables beneficios para la vida personal, familiar y profesional. Nos referimos a lo que podemos denominar el aprovechamiento del tiempo o la posibilidad de disponer de mayor cantidad y calidad de tiempo. El reparto del tiempo siempre ha sido una variable que ha influido en las desigualdades entre mujeres y hombres, con el obstáculo actual de la conciliación de la vida personal y familiar con la actividad profesional.

Buena muestra de la desigualitaria gestión del tiempo son los datos objetivos; así, de las 24 horas que tiene el día, las mujeres dedican un menor número a ellas mismas, es decir, a su persona, al ocio e, incluso, a su trabajo fuera de lo doméstico, y, por el contrario, más horas que ellos al cuidado de la familia y a las labores domésticas. El tiempo no vale lo mismo para unas y $u_{n o s}{ }^{38}$. Las tecnologías pueden facilitar las cosas y si se saben aplicar redundan en un ahorro de tiempo al ofrecer alternativas para desarrollar tareas sin necesidad de desplazamientos y en menor tiempo. En este sentido, se convierten en aliadas femeninas que para el trabajo, formarse, entretenerse, arreglar citas, papeleos, etc., ganan tiempo valioso que dedicarán a otras tareas.

De una observación de cómo son los accesos, usos y presencias de las mujeres en Internet, resulta posible establecer un perfil de las mujeres usuarias que se traza atendiendo a datos como la edad, estudios y situación laboral. Con los datos del CIS (2001) el perfil más frecuente es el de una usuaria joven, menor de 35 años, con estudios universitarios medios o superiores, mientras que las mujeres más jóvenes acceden con menos frecuencia ya sea

38. Sin ir más lejos, el 3 de diciembre de 2008, la prensa española se hacía eco de los datos publicados en el Estudio «En torno a la familia española: Análisis y reflexiones desde Perspectivas Sociológicas y Económicas», de la Fundación de Cajas de Ahorro (FUNDAS). Así, se traduce en números la dedicación de las españolas a las tareas domésticas, pues dedican el triple de tiempo que los hombres a este trabajo que viene a ser un total de 4:55 horas al día mientras que los hombres sólo dedican una media de 1:37 horas al día. Es España junto a Italia, el país de la Unión Europea donde hay mayor diferencia en lo que se refiere al reparto del trabajo doméstico, muy alejada de otros países como Suecia, Noruega o Reino Unido en los que el reparto es más equitativo (Consultar www.elpais.com, sección Sociedad, última consulta 3/12/2008). 
por su menor inserción laboral o por las dificultades de las estudiantes para acceder diariamente a Internet. Por lo que se refiere al uso principal, nos encontramos con que la actividad más frecuente es la lectura de noticias, siendo las usuarias con estudios medios o superiores aquéllas que buscan más información y documentación, siendo menos frecuente realizar gestiones. Las mujeres más jóvenes, junto a las que tienen menor nivel de estudios, realizan otras actividades como juegos, descargas de música o mandar mensajes a teléfonos móviles. La imagen que las mujeres tienen de sí mismas coincide con el perfil de usuaria, se ven como mujeres activas, trabajan remuneradamente, son jóvenes y urbanas. Sin embargo, otras condiciones sociales como ser ama de casa, tener más de 45 años y vivir en un entorno rural, son elementos que reducen considerablemente las posibilidades de acceder a las tecnologías.

De un estudio realizado sobre las características de las participantes en DigitalEve (organización global de mujeres a favor de las mujeres que se relacionan entre ellas exclusivamente a través de Internet) se extrae que la mayoría de estas infoconectadas trabajan en sectores y empleos relacionados con las TICs, los medios de comunicación y tecnologías digitales, integrándose mujeres de todas las edades, niveles educativos y cualificación tecnológica, si bien todas ellas comparten su interés por las TICs.

Por tanto, aun afirmando la diversidad apreciada entre todas las participantes, la autora constata ciertos puntos en común entre todas ellas. Así, les gustan las tecnologías, por lo que no son «tecnofóbicas», y les gustan por las enormes posibilidades que les ofrecen por lo que reconocen su «interés tecnológico» aunque mediatizado por su interés laboral, político o social. Por tanto, aun cuando trabajan en el ámbito de la tecnología, vienen redireccionadas de otros ámbitos profesionales de lo más diverso. Todas ellas están de acuerdo en criticar que los hombres construyan una cultura que valora excesivamente la cualificación tecnológica, porque es un factor que excluye a muchas mujeres que, aunque no sobresalen por sus habilidades tecnológicas, tienen otras igual de valiosas o incluso más. Precisamente, esta exclusión femenina es la causa que desilusiona a muchas mujeres y propicia que den la espalda a las tecnologías eludiendo su implicación. Esto se cumple en muchas de las mujeres integrantes de la Red que son autodidactas, habiendo adquirido sus habilidades y conocimientos tecnológicos con libros, con amistades o simplemente experimentando y aprendiendo de sus propios errores cometidos (un aprendizaje sin duda meritorio, pero que no tiene reconocimiento en el mercado, que valora negativamente la ausencia de formalidad en el aprendizaje).

Hemos querido cerrar este epígrafe sobre la presencia femenina en la Red dando a conocer la existencia de esta Red de mujeres que rompe con todos 
los estereotipos de género al estar integrada por mujeres preocupadas por los usos y aplicaciones tecnológicas y no tanto por el dominio tecnológico.

\section{Alternativas para detener la brecha}

Esta tajante separación social establecida en base a la alfabetización tecnológica, y que se agrava si se tiene en cuenta la variable de "género», tiene que abordarse desde una perspectiva de género para ponerle coto. Como bien señala Cecilia Castaño, «la tecnología no es discriminatoria. Es la voluntad humana. No basta con que las escuelas tengan un ordenador por cada dos niños; hay que cambiar la cultura de la tecnología. Cuando se habla de Internet y la sociedad de la información se evalúa el número de ordenadores por cada 100 habitantes o el porcentaje de población que usa Internet y compra por este medio. Se olvida, sin embargo, la variedad, las diferencias y las desigualdades existentes. Quienes se comunican son personas que usan la tecnología para informarse, para trabajar, para contactar con amigos y familiares o para comprar. Lo realmente importante es qué hace la gente, cómo lo hace y si sabe hacerlo bien ${ }^{39}$. Si atendemos a la posición de las mujeres como agentes activos de construcción de la Sociedad de la Información, comprobamos que están 10 puntos por debajo de los hombres, por lo que no hay una equilibrada presencia femenina.

Así las cosas, si partimos de la premisa incuestionable de que, a día de hoy, el acceso y el aprendizaje tecnológico tiene que ser igual para hombres y mujeres tanto en lo que se refiere al tiempo, a los recursos y al empleo:

- Hay que analizar la dificultades concretas del acceso de las mujeres a las TICs introduciendo un sistema de indicadores sobre el acceso, la frecuencia, la duración, los usos, etc., que sean útiles para orientar las políticas públicas e incentivar a las empresas e instituciones privadas.

- y, en consecuencia, aportar soluciones específicas y adecuadas para superar la menor integración femenina en la Sociedad de la Información (el tiempo, los recursos y el empleo de las tecnologías no es el mismo entre hombres y mujeres) incentivando investigaciones que sirvan para detectar las diferencias en los colectivos de mujeres en su participación en la Sociedad de la Información (profesorado, alumnado, empresarias, profesionales, trabajadoras, etc.).

- Para solucionar el problema del tiempo que sufren las mujeres, hay que adaptar las herramientas y los contenidos (tengamos en cuenta que las

39. www.elpais.es. Reportaje «Sociedad de personas, no de tecnologías» publicado el 19/01/2006, última consulta 13/03/2006. 
mujeres confiesan que...). Así pues, una demanda femenina atiende a la eficacia y a la utilidad concreta que es lo que realmente buscan las mujeres en las TICs. No obstante lo anterior, resulta imprescindible que las mujeres tengan la posibilidad de liberar otros tiempos, esto es, que se adopten medidas efectivas dirigidas a conciliar la vida personal familiar y la vida laboral buscando la motivación y ofrecer utilidades para conciliar y hacer el sistema más democrático. Y ello sin olvidar que las tecnologías permiten un cambio de las relaciones, pues éstas pueden convertirse en un instrumento útil para ejercer la corresponsabilidad en cuanto que pueden facilitar tanto el trabajo profesional realizado fuera de casa como el trabajo doméstico.

- En este orden de cosas, el problema de los recursos se detecta en el momento inicial de la alfabetización, pero también para el uso continuado de las tecnologías, por lo que una medida efectiva consistirá en ofrecer ayuda para adquirir equipos informáticos a las mujeres y a los grupos en que se integran, así como un reciclaje continuado.

Por lo que se refiere a las infraestructuras, no podemos dejar de hacer constar que el 39\% de las mujeres que no usan Internet es porque no tienen ordenador ni pueden acceder a uno, ya sea por su precio elevado o porque no pueden hacer frente a las constantes innovaciones. Se pone de manifiesto igualmente, que las aplicaciones informáticas no están pensadas ni diseñadas para las mujeres porque, en general, no mejoran su calidad de vida. Además el idioma es una barrera evidente, el handicap del inglés, por no nombrar que escasean los contenidos dirigidos a las mujeres como protagonistas, como sujetos y no simples objetos de consumo masculino; además, los elevados costes no ayudan, si tenemos en cuenta que España es el país más caro de la Unión Europea y que, como está más que constatado, la economía de las mujeres, en general, es bastante peor que la de los hombres.

- En cuanto al empleo, sin ningún género de dudas, la alfabetización digital capacita profesionalmente y mejora el acceso laboral y la remuneración, además las tecnologías han de servir como herramientas de progreso y promoción y para ayudar a otras mujeres desfavorecidas

\section{Propuestas de actuación para construir una sociedad de la información} desde la igualdad:

- sensibilización de todos los sectores sociales sobre la necesidad de construir una sociedad inclusiva y democrática: educación y formación desde la igualdad entre mujeres y hombres.

- potenciar la participación de las mujeres en el ámbito tecnológico. 
- inclusión femenina en los medios de comunicación para alcanzar la representación y difusión de una pluralidad de intereses.

- compromiso entre los grupos de mujeres para trabajar juntas y aunar fuerzas. 